Electronic Supporting Information

\title{
DNA Strand Replacement Mechanism in Molecular Beacons Encoded for Detection of Cancer Biomarkers
}

\author{
Magdalena Stobiecka ${ }^{\mathrm{a} *}$ and Agata Chalupa ${ }^{\mathrm{b}}$ \\ ${ }^{a}$ Department of Biophysics, Warsaw University of Life Sciences (SGGW), 02776 Warsaw, \\ Poland \\ ${ }^{b}$ Institute of Nanoparticle Nanocarriers, 11010 Barczewo, Poland
}

* Corresponding Author._E-mail address: magdalena_stobiecka@sggw.pl

Phone: +48.22 .593 .8621 .

Fax: +48.22 .593 .8619 . 


\section{MATERIALS AND METHODS}

Chemicals. The following 30-mer SurMB and 20-mer target $t$ DNA sequences have been used in experiments:

SurMB: 5'-6-FAM-CCT GGC CCA GCC TTC CAG CTC CTT GCC AGG-Dabcyl$3^{\prime}$,

tDNA: 5'-CAA GGA GCT GGA AGG CTG GG-3',

where underline indicates the stem area bases, boldface marks the antisense sequence, 6-FAM is the fluorescence dye 6-carboxyfluorescein, and Dabcyl is the dark quencher 4,4-dimethylamino-azobenzene-4'-carboxylic acid. They were synthesized by the Laboratory of DNA Sequencing and Oligonucleotides Synthesis, Institute of Biochemistry and Biophysics of the Polish Academy of Sciences (IBB PAS, Warsaw, Poland). These oligonucleotides were purified and tested by HPLC. SurMB loop was specifically coded to target nucleotides 232-251 of the 1916-bp survivin mRNA (Homo sapiens baculoviral IAP repeat-containing 5 (BIRC5)), found by Olie et al. ${ }^{1}$ to be the most efficient for BIRC5 gene silencing. Morpholinepropanesulfonic acid (MOPS) and sodium nitrate $\left(\mathrm{NaNO}_{3}\right)$ were obtained from Sigma-Aldrich Chemical Company (St. Louis, MO, USA). All chemicals were of analytical grade purity. Aqueous solutions were prepared with freshly deionized water with $18.2 \mathrm{M} \Omega \mathrm{cm}$ resistivity (Hydrolab, Wiślina, Poland). All concentrations of added reagents cited in this paper are final concentrations obtained after mixing, unless otherwise noted. 
Instrumentation. The fluorescence spectra were recorded using Spectrometer model LS55 (Perkin Elmer, Waltham, MA, USA), with $20 \mathrm{~kW}$ Xenon light source and a photomultiplier tube detector. During the MB melting experiments, the temperature was scanned stepwise, with step height of $2 \mathrm{deg}$, and measurements were performed after 1 min waiting at each temperature. The excitation and emission slit widths were set to $5.0 \mathrm{~nm}$ with scan speed $500 \mathrm{~nm} / \mathrm{min}$. The measurements were performed in $10 \mathrm{mM}$ MOPS buffer $+50 \mathrm{mM} \mathrm{NaNO}_{3}$ solutions, $\mathrm{pH}$ 7.45. The excitation and emission wavelength were set to $\lambda_{\mathrm{ex}}=480 \mathrm{~nm}$ and $\lambda_{\mathrm{em}}=516 \mathrm{~nm}$, respectively.

\section{Calculation of equilibrium concentrations of conformational polymorphs of SurMB}

Polymorph formation reactions:

$\begin{array}{lll}\mathrm{MB}_{\text {(open) }} & =\mathrm{MB}_{\text {(closed,1) }} \\ \mathrm{c}_{\text {open }} & \mathrm{c}_{1}\end{array}$

Equilibrium constants:

$\mathrm{K}_{1}=\mathrm{c}_{1} / \mathrm{c}_{\text {open }}, \mathrm{K}_{2}=\mathrm{c}_{2} / \mathrm{c}_{\text {open }}, \mathrm{K}_{3}=\mathrm{c}_{3} / \mathrm{c}_{\text {open }}$

Equations:

$\mathrm{c}_{1}=\mathrm{c}_{\text {open }} \mathrm{K}_{1}, \mathrm{c}_{2}=\mathrm{c}_{\text {open }} \mathrm{K}_{2}, \mathrm{c}_{3}=\mathrm{c}_{\text {open }} \mathrm{K}_{3}$

$\mathrm{c}_{\text {tot }}=\mathrm{c}_{\text {open }}+\mathrm{c}_{1}+\mathrm{c}_{2}+\mathrm{c}_{3}$

$c_{\text {tot }}=c_{\text {open }}+c_{\text {open }} \mathrm{K}_{1}+c_{\text {open }} \mathrm{K}_{2}+\mathrm{c}_{\text {open }} \mathrm{K}_{3}=\mathrm{c}_{\text {open }}\left(1+\mathrm{K}_{1}+\mathrm{K}_{2}+\mathrm{K}_{3}\right)$

$\mathrm{c}_{\mathrm{open}}=\mathrm{c}_{\mathrm{tot}} /\left(1+\mathrm{K}_{1}+\mathrm{K}_{2}+\mathrm{K}_{3}\right)$

First, the concentration of the open form of $\mathrm{MB}$ is calculated using eq. (5). Then, the concentrations of other polymorphs are calculated using relations (2). 


\section{Kinetics of target-binding}

The solution of the full kinetics of the reaction system:

$\mathrm{MB}+\mathrm{tDNA} \underset{k_{b}}{\stackrel{k_{f}}{\rightleftarrows}}$ MB.tDNA

B T D

taking into account also the rate of the backward reaction was presented by Tsourkas et al. ${ }^{2}$, as follows:

$1-\frac{D_{t}}{D_{e q}}=\exp \left\{-\Delta k_{f} t\right\}\left(1-\lambda \frac{D_{t}}{D_{e q}}\right)$

where $D_{\mathrm{t}}$ and $D_{\text {eq }}$ are the concentrations of the duplex at time $t$ and at equilibrium, respectively,

$\Delta=\sqrt{\left(B_{0}+T_{0}+K\right)^{2}-4 B_{0} T_{0}}$

$D_{\text {eq }}=1 / 2\left(B_{0}+T_{0}+K-\Delta\right)$

$\lambda=D_{\mathrm{eq}}{ }^{2} /\left(B_{0} T_{0}\right)$

Since

$\frac{D_{t}}{D_{e q}}=\frac{F_{t}-F_{0}}{F_{e q}-F_{0}}$

where $F_{\mathrm{t}}, F_{\mathrm{eq}}$, and $F_{0}$, are the fluorescence at time $t$, at equilibrium, and the initial fluorescence, respectively, the experimental function $\left(F_{\mathrm{t}}-F_{0}\right) /\left(F_{\mathrm{eq}}-F_{0}\right)$ can be fitted directly by equation (7). However, the equilibrium data are necessary to follow this procedure, so the reaction must be completed during the experiment which may take several hours.

The kinetic data treatment can be substantially simplified when the system is highly irreversible, so that the backward reaction rate can be neglected. Since, in fact, the target 
binding process by $\mathrm{MB}$ is strongly irreversible, we have applied this simplification in the following derivation of the kinetic data treatment.

The kinetic analysis is based on fitting of experimental data with a second order kinetic equation describing the reaction:

SurMB $+t$ DNA $\rightarrow$ SurMB- $t$ DNA

$c_{\mathrm{MB}} \quad c_{\text {trg }} \quad c_{\text {complex }}$

For this irreversible reaction, the rate $v$, at a constant temperature, is given by:

$v=\left(\frac{\partial c_{\text {complex }}}{\partial \tau}\right)_{T}=k_{T}\left(c_{0, M B}-c_{\text {complex }}\right)\left(c_{0, \text { trg }}-c_{\text {complex }}\right)$

where $k_{\mathrm{T}}$ is the temperature dependent reaction rate constant, $c_{0, \mathrm{MB}}$ and $c_{0, \operatorname{trg}}$ are the initial concentrations of SurMB and target, respectively, $\tau$ is the time, and $c_{\text {complex }}$ is the concentration of the reaction product. This equation can be simplified for the case: $c_{0, \mathrm{MB}}=$ $c_{0, \operatorname{trg}}=c_{0}$. After the separation of variables, one obtains:

$\frac{\partial c_{\text {complex }}}{\left(c_{0}-c_{\text {complex }}\right)^{2}}=k_{T} \partial \tau$

This equation can be easily integrated:

$\int_{0}^{c} \frac{\partial c}{\left(c_{0}-c\right)^{2}}=\int_{0}^{t} k_{T} \partial \tau$

where $c=c_{\text {complex. }}$. Substituting $z=c_{0}-c$, one obtains: $\mathrm{d} z=-\mathrm{d} c$ and:

$-\int_{0}^{c_{0}-c} \frac{\partial z}{z^{2}}=\int_{0}^{t} k_{T} \partial \tau$ 
The integration yields:

$$
\frac{1}{\left(c_{0}-c\right)}-\frac{1}{c_{0}}=k_{T} \tau
$$

After rearrangement, the following function describing the progression of the targetstimulated MB dissociation is obtained:

$$
c=c_{0}\left(1-\frac{1}{\left(1+c_{0} k_{T} \tau\right)}\right)
$$

This equation represents a single wave with monotonic derivative. If the reaction would proceed via two separable steps, a two wave function would be expected. To apply the obtained solution for one step reaction to the analysis of experimental data, the following expression for fluorescence emission intensity as a function of SurMB- $t$ DNA complex concentration has been assumed:

$F=F_{1}+F_{2}=\varepsilon_{\text {off }}\left(c_{0}-c\right)+\varepsilon_{\mathrm{on}} c$

where the first term $\left(F_{1}\right)$ corresponds to the residual fluorescence from the SurMB in the closed conformation and the second term $\left(F_{2}\right)$ corresponds to the fluorescence of the SurMB-tDNA duplex; $\varepsilon_{\mathrm{i}}$ is the fluorescence coefficient for species $i$ :

$\varepsilon_{i}=\left(\frac{\partial F}{\partial c_{i}}\right)$

Combining equations (18) and (19), one obtains:

$$
F=\varepsilon_{o f f} c_{0}+\left(\varepsilon_{o n}-\varepsilon_{o f f}\right) c_{0}\left[1-\frac{1}{\left(1+c_{0} k_{T} \tau\right)}\right]
$$

or: 
$F=F_{i n i}+\left(F_{\max }-F_{i n i}\right)\left[1-\frac{1}{\left(1+c_{0} k_{T} \tau\right)}\right]$

where $F_{\text {ini }}$ is the initial fluorescence and $F_{\max }$ is the fluorescence of pure SurMB-tDNA duplex at the concentration $c_{0}$.

Equation (22) can be employed for data fitting and determination of the rate constants $k_{\mathrm{T}}$ using simplex iterations.

\section{References}

1. R. A. Olie, A. P. Simoes-Wust, B. Baumann, S. H. Leech, D. Fabbro, R. A. Stahel, U. Zangemeister-Wittke, Cancer Res. 2000, 60. 2805-2809.

2. A. Tsourkas, M. A. Behlke, S. D. Rose, G. Bao, Nucl. Acids Res. 2003, 31. 1319-1330, DOI: doi: 10.1093/nar/gkg212. 